\title{
El discurso en torno a la sexualidad: Comparación entre dos libros de orientación para adolescentes
}

\section{The discourse on sexuality: a comparison of two sex orientation books for adolescents}

DOI: https://doi.org/10.32870/dse.v0i21.641

\section{Mariana Guadalupe Molina Fuentes*}

\begin{abstract}
Resumen
La educación sexual es sin duda uno de los rubros más importantes por cubrir en nuestro país en la actualidad. Si bien parece haber un consenso generalizado al respecto, lo cierto es que las formas de aproximarse y de impartir los contenidos de esa educación son tan diversas como los grupos que conforman el tejido social. En este artículo se hace un análisis del discurso subyacente en dos libros cuyo objetivo consiste en orientar a las y los adolescentes en el ejercicio de su sexualidad. Uno de ellos fue editado por el gobierno del entonces Distrito Federal; el otro por la organización católica Red Familia. En este ejercicio, de ningún modo se pretende establecer juicios de valor respecto de su contenido, sino señalar sus similitudes y diferencias en el uso del lenguaje, la construcción de sus argumentos, y el modo de entender la sexualidad.
\end{abstract}

Palabras clave: educación sexual - sexualidad - análisis del discurso - Red Familia - Gobierno del Distrito Federal.

\begin{abstract}
Sex education is undoubtedly one of the most important items to cover in our country today. Although there seems to be general consensus on the matter, the truth is that the ways of approaching and teaching the contents of that education are as diverse as the groups that make up the social fabric. This article analyzes the underlying discourse in two books whose objective is to guide adolescents in the exercise of their sexuality. One of them was published by the local government of Mexico City, and the other by the Catholic organization Red Familia ("Family Network"). This exercise is not intended to make any value judgments regarding such books, but only to point out their similarities and differences in the use of language, the construction of their arguments, and their ways of understanding sexuality.
\end{abstract}

Key words: sex education - sexuality - discourse analysis - Family Network - Federal District Government.

* Doctora en Ciencia Social con especialidad en Sociología. Investigadora Nivel 1 del Sistema Nacional de Investigadores, coordinadora de la Cátedra Extraordinaria "Benito Juárez", en el Instituto de Investigaciones Jurídicas de la UNAM. México. mmolina@unam.mx 


\section{Introducción}

La educación sexual ha sido un tema de profundos debates en México, al menos desde la década de 1970, cuando el gobierno del entonces presidente Luis Echeverría Álvarez (1970-1076) lanzó una campaña de planificación familiar que desafiaba algunos principios de la moral católica. ${ }^{1}$ Hoy, a más de cuatro décadas de distancia, los contenidos y la forma en la que se aborda la sexualidad en los libros de nivel básico son todavía objeto de discusión. Las discrepancias entre los grupos que defienden un modelo educativo laico y los que proponen el apego a los valores religiosos son visibles a través de sus discursos, en los que pueden apreciarse posiciones claras, no solo con respecto a la sexualidad sino a una forma de concebir el bienestar personal y social.

El objetivo en este artículo consiste en comparar la construcción del discurso en dos libros de educación sexual a partir de las aportaciones analíticas de autores como Benveniste (1977), Austin (1982), Peirce (1987), e Irvine y Gal (1995), entre otros. Para ello se propone un esquema comparativo desde tres dimensiones: $a$ ) las condiciones de producción de los textos; $b$ ) su filiación ideológica; y c) la intención de formar posiciones morales específicas entre sus destinatarios. Es importante subrayar que aquí de ninguna manera se pretende evaluar la pertinencia, la validez o la supremacía de alguno de estos discursos, sino aprehender sus similitudes y sus distintivos. Así pues, el artículo se estructura en tres partes. En la primera se describe brevemente el contexto espacio-temporal en el que se editaron los libros analizados, ubicando además a quienes emiten el discurso. Luego, se procura desentrañar los valores subyacentes en ambos textos, así como su manifestación a través de: $a$ ) sus contenidos y la forma de abordarlos; $b$ ) la multiplicidad de voces que incluyen; $y c$ ) el vínculo que se construye, mediante el lenguaje, con las y los destinatarios de los libros. Por último, se ofrecen algunas observaciones que resultan del análisis.

\section{Condiciones de producción de dos libros sobre educación sexual}

En 2008 se editaron los libros Sexualidad y salud humana, y Tu futuro en libertad, dos materiales cuyo propósito explícito consistía en complementar los contenidos de los libros de texto de biología y en orientar a las y los jóvenes para reflexionar sobre el ejercicio de su propia sexualidad. En términos generales, puede decirse que ambos nacieron a partir de una misma preocupación; sin embargo, los discursos que estructuran sus contenidos son profundamente distintos.

Peirce (1987) y Levinson (1983) sostuvieron que el análisis de cualquier discurso debe realizarse, necesariamente, en función del contexto en el que se produce. Bajo esa lógica, en este apartado se exponen las condiciones en las que se pusieron en circulación los libros aquí referidos. Para ello se ubica también a los autores y sus posiciones morales, en el entendido de que

1 Este punto resultó problemático, porque en las corrientes más tradicionales del catolicismo se sostiene que el acto sexual tiene una función preminentemente reproductiva. De ahí se deriva el rechazo al uso de anticonceptivos, central para la planificación familiar.

Diálo@os sobre Educación 
los materiales iban dirigidos a estudiantes de secundaria y de preparatoria.

En su Obra lógico-semiótica, Peirce (1987) estableció que la asignación de significados no es un hecho único sino un proceso infinito de interpretación. ${ }^{2}$ Ese argumento es valioso para el presente análisis porque evidencia dos cosas. La primera, que la interpretación de un discurso no es rígida; es decir, que el análisis de las palabras y sus definiciones no es suficiente para comprender un texto porque este no es un producto autocontenido. Dicho de otra forma, los significados de un discurso están siempre en función del contexto en el que se produce y se distribuye. Y la segunda, que en un contexto en particular esas palabras pueden pensarse como índices ${ }^{3}$ o señales de una posición social, ideológica o del tipo de relación que existe entre quienes participan del acto comunicativo (Irvine y Gal, 1995).

\section{Los creadores del discurso y el interés por la educación sexual}

Como apunta Foucault (1977), la sexualidad en la cultura occidental estuvo sujeta a la regulación del cristianismo y a una consecuente censura, cuando menos hasta el siglo XVII. La idea de que el tema habría de permanecer oculto se desvaneció, para dar paso a la circulación de discursos en los que la sexualidad ocupa un papel central. Empero, ello no significa que esté exenta de controles y de normas. Aún en nuestros días, para la Iglesia católica es un tema crucial, porque a través de ella se cumple con una función reproductiva. En consecuencia, las prácticas que no se vinculan con la reproducción suelen calificarse de inmorales, prohibidas, o cuando menos desviadas. A ello se suma la regulación por parte del aparato estatal, que problematiza la sexualidad como un asunto de salud pública a través de instituciones laicas como la ciencia y la jurisprudencia, entre otras.

En México, el interés por la sexualidad y su regulación puede entenderse si se consideran algunas tendencias de las últimas décadas: la tasa de nupcialidad se redujo de $74 \%$ a $50 \%$ entre 1990 y 2010; el porcentaje de población infectada con VIH es de 0.3, y en la Ciudad de México 54\% de las madres se embarazó por primera vez antes de los 19 años (INEGI, 2013).

Estos datos ponen de manifiesto la necesidad de brindar una educación sexual oportuna, cuyo espacio natural parece ser la escuela. Pero el acuerdo sobre la pertinencia de introducir ese tema en el currículum educativo se resquebraja ante la contraposición de grupos que ostentan nociones distintas sobre la forma en la que habría de entenderse, enseñarse y regularse la sexualidad. Para quienes editan los materiales educativos que se analizan en este artículo, el contenido de los libros de texto distribuidos por el gobierno federal es insuficiente.

Por un lado se encuentra el gobierno del entonces Distrito Federal (GDF), cuyos represen-

2 Este argumento contradice el supuesto del estructuralismo saussuriano, según el cual la relación entre significado y significante se mantiene estable.

3 Peirce define como índices a aquellos signos que indican o que mantienen una relación con el objeto que refieren. En ese tenor, las palabras pueden considerarse índices de una posición social, política o ideológica de quien las usa. 
tantes actúan bajo la premisa de que la educación sexual es un medio para evitar problemas de carácter público, tanto en el ámbito sanitario como en el de la convivencia entre ciudadanos con identidades, preferencias y prácticas diversas. Por su parte, la Red Familia es una organización de la sociedad civil, católica, y no adscrita a la estructura eclesial. Sus integrantes conciben la educación sexual como un medio para promover instituciones como la vida, los derechos humanos, el matrimonio y la familia.

A partir de esta descripción puede advertirse que los dos actores muestran un profundo interés en el espacio educativo como medio de regulación de la sexualidad. No obstante, se diferencian discursivamente en virtud de la posición ideológica que mantienen:

El principio de laicidad ${ }^{4}$ postula el respeto a la libertad de conciencia, la autonomía de lo político frente a lo religioso, y la igualdad de los individuos y sus asociaciones ante la ley (Blancarte, 2008). Quienes editaron el libro del GDF parecen entender el bienestar como aquella situación en la que toda persona está en condiciones de desarrollarse plenamente, en el marco de la ciudadanía y de la igualdad de derechos que garantiza el Estado.

Por su parte, el integrismo ${ }^{5}$ católico sostiene que la moral religiosa debería permear tanto el espacio privado como el público. Con una ideología ${ }^{6}$ anclada en la tradición, la Red Familia sostiene que el bienestar personal y social se basa en la dignidad de la persona humana. Para mantenerla, el Estado debe asegurar la protección de la familia, entendida como la unión de un hombre y una mujer a través del matrimonio abierto a la vida (Red Familia, 2012).

Las posiciones aquí referidas constituyen la base de los discursos tanto en Tu futuro en libertad como en Sexualidad y salud humana, dos libros de corte educativo que, como se explicará en el siguiente acápite, se pusieron en circulación en un contexto especialmente tenso.

\section{El Gobierno del Distrito Federal y la amenaza al orden tradicional}

Durante las administraciones de Alejandro Encinas y de Marcelo Ebrard como Jefes de Gobierno, se desató una profunda polémica alrededor de dos modificaciones a la legislación. En 2006 se aprobó la Ley de Sociedades de Convivencia. En ella se reglamentaron los derechos y obligaciones de los miembros de hogares que carecen de lazos consanguíneos o conyugales, entre cuyos beneficiarios se encontraron las parejas del mismo sexo. Al año siguiente, el Distrito Federal se convirtió en la primera entidad federativa en despenalizar el aborto en México. Las reacciones a estas disposiciones legales estuvieron polarizadas: lo que para unos reflejaba el progreso en materia de política social, para otros representó un salto en detrimento de la esta-

4 Es decir, la autonomía del Estado respecto de las normas, instituciones y autoridades dogmáticas.

5 Se entiende por integrismo al modelo que coloca la religión como eje de la organización social, y por lo tanto como rectora de las prácticas individuales y sociales.

6 En este artículo nos referiremos como ideología al conjunto de valores, ideas, objetivos y nociones morales que brindan un sentido al modo en que los sujetos se conciben a sí mismos y comprenden su entorno social.

Diólo pos sobre Educación año 11 | número 21 | julio-diciembre 2020 | ISSN 2007-2171 
bilidad anclada en los valores tradicionales. ${ }^{7}$

El clima de tensión generado por la aprobación de estas leyes se ubica como el telón de fondo de la redacción, edición, y distribución de los libros que se analizan en este artículo. Aquí se sugiere que ambos pueden pensarse como una respuesta al debate público de la época, y que en su discurso pueden identificarse tanto sus posicionamientos como algunas referencias a sus contrincantes.

Por ejemplo, en Tu futuro en libertad se afirma que algunas organizaciones religiosas controlan la sexualidad de sus fieles, pero no pueden hacerlo en términos legales porque el Estado es laico. Este fragmento puede entenderse como una respuesta a las declaraciones de grupos conservadores sobre las leyes antes citadas, calificadas como actos reprobables debido a su ruptura con los valores que, desde esa perspectiva, mantienen la cohesión y la estabilidad social. Por su parte, y a modo de reivindicación de las posiciones que mantienen la Red Familia y otras organizaciones asociadas, ${ }^{8}$ en Sexualidad y salud humana se refiere que "la educación sexual corresponde en primer lugar a la familia, y debe estar de acuerdo con sus principios, costumbres y valores" (Fernández, 2008: 27).

Como se discutirá en los siguientes apartados, la carga ideológica de los textos puede rastrearse mediante el uso de palabras que supuestamente describen una realidad objetiva, pero que tienen un fuerte componente normativo. Así pues, los libros que aquí se analizan tienen un doble propósito: ofrecen información sobre ciertos temas y buscan crear posiciones morales sobre ellos.

\section{El discurso y la ideología subyacente en los libros de educación sexual}

El objetivo en este acápite consiste en explorar el discurso que contienen los libros mencionados. Para ello, se realizará un ejercicio comparativo en torno a tres dimensiones: los contenidos y la forma en la que se abordan; la multiplicidad de voces que coexisten en el discurso de cada uno; y el uso del lenguaje en el vínculo con sus destinatarios.

\section{Formas de pensar en la sexualidad}

Una primera estrategia para acercarse a los textos aquí estudiados, consiste en revisar sus contenidos a través de sus índices. Si bien los temas abordados en los libros son insuficientes para hacer un análisis del discurso, lo cierto es que su organización da cuenta de la estructura con base en la cual se construye. En este punto cabe recordar que ambos son de corte educativo,

\footnotetext{
7 En esa coyuntura se pusieron en circulación una multiplicidad de discursos. Algunos ejemplos de ello son el resurgimiento de la defensa por la diversidad sexual, las organizaciones a favor de los derechos humanos, los pronunciamientos antiaborto de asociaciones como ProVida o la Red Familia, y múltiples demandas de una educación de calidad por parte de grupos como la Red por la Educación o la Confederación Nacional de Escuelas Particulares.

8 Algunos ejemplos son la Unión Nacional de Padres de Familia (UNPF) y la Asociación de Laicos para la Madurez Afectiva y Sexual (ALMAS).
}

Diálo@os sobre Educación 
que van dirigidos a adolescentes, y que están pensados como complementarios a los libros de texto gratuitos que distribuye el gobierno federal. En esa lógica, cumplen en principio con una función informativa, y como tal se aproximan a una definición de sexualidad, sus prácticas, sus riesgos y la forma de prevenirlos. A primera vista, estas características compartidas podrían conducir a la hipótesis de que la estructura de ambos discursos es similar; sin embargo, es notorio que existen diferencias profundas entre ellos. Como se argumentará posteriormente, esas distinciones pueden pensarse a partir del posicionamiento ideológico. Además, este no se limita a la descripción de hechos o de eventos sino que adquiere un carácter normativo.

El texto de Sexualidad y salud humana comienza por abordar las potencialidades del acto sexual a partir de la reproducción y de los vínculos afectivos; luego, da paso a la responsabilidad, la salud y los métodos de prevención, e incluye un último apartado sobre planificación familiar. Así, en su discurso se enfatizan las funciones de la sexualidad como parte de la especie humana y no de la vida individual, al tiempo que los derechos humanos se vinculan con la dignidad de la persona y no con el ejercicio de la ciudadanía. El último apartado es también sintomático de la ideología que subyace en el texto, pues se le otorga un papel preponderante a la familia como unidad social.

Por el contrario, Tu futuro en Libertad inicia su aproximación desde el individuo, sus relaciones personales y la diversidad. En el segundo apartado se brinda una definición del concepto de sexualidad, y en el siguiente se informa sobre las prácticas sexuales, sus riesgos (infecciones, enfermedades y embarazos no deseados) y las formas de prevenirlos. Finalmente, el libro incluye una sección sobre los derechos humanos, los derechos sexuales y la laicidad del Estado. El orden a partir del cual se construye el discurso brinda ya algunas pistas sobre la adscripción ideológica del texto: el individuo ocupa un papel central en este libro, en el que la diversidad y la experimentación sexual se entienden como parte de los derechos que el Estado ha de garantizar.

El contenido de los libros que aquí se analizan es extenso, y debe reconocerse que este breve ejercicio no agota toda la riqueza de su discurso. Tomando en cuenta esta limitante, se propone sistematizar el análisis a partir de tres interrogantes: a) ¿qué es la sexualidad?; b) ¿cómo se ejerce?; y c) ¿quién o quiénes se legitiman como autoridades para brindar orientación al respecto?

Con el objetivo de mostrar las similitudes y diferencias entre ambos libros, se recurrirá a citas textuales. Algunas palabras han sido subrayadas por quien escribe este artículo, puesto que se identifican como índices (o indicativos) de la ideología del discurso analizado.

a) ¿Qué es la sexualidad? El objetivo primordial de los libros que se analizan en este artículo consiste en brindar educación sexual. Por ese motivo, lógicamente, el concepto de sexualidad es crucial para ambos. Como se anotó con anterioridad, la forma de construir lo que esta significa es esencialmente distinta en cada uno de ellos.

Diólo@os año 11 | número 21 | julio-diciembre 2020 | ISSN 2007-2171 
En el libro editado por la Red Familia puede leerse que es "[...] la integración de varias potencialidades de los seres humanos: la capacidad de amar y de relacionarse con los demás; la de dar y recibir placer; la posibilidad de decidir y desarrollarse social y profesionalmente y la de reproducirse" (Fernández, 2008: 10). Inmediatamente después de esta definición se incluyen dos subapartados, en los que se explica la sexualidad como potencia de reproducción y como lugar de comunicación física y afectiva.

Es notable que la capacidad de amar aparezca como la primera de las potencialidades enunciadas en este fragmento, y que el concepto se construya en función de "los demás". Más que como un ejercicio de la individualidad, el libro presenta la sexualidad como una relación; un vínculo con otros a partir del amor, del placer, del desarrollo o de la reproducción. Asimismo, las aclaraciones que siguen al concepto subrayan la dimensión reproductiva por encima de las otras.

A decir de Irvine y Gal (1995), las palabras constituyen un índice en la medida en que remiten a una ideología, un compromiso político, un posicionamiento social o a un esquema de pensamiento respecto del modo en el que funciona el mundo. En el párrafo antes citado es posible identificar varias palabras cuyo uso puede vincularse con la doctrina católica: el texto no se refiere a individuos ni a ciudadanos, sino a seres humanos capaces de amar, que mantienen un lazo con los demás y que tienen la posibilidad de reproducirse. Es necesario recordar que uno de los principios básicos de la doctrina social católica es el amor, que genera cohesión social frente a la individualización que se originó en la era moderna. El catolicismo se posiciona en contra de esta tendencia, y no se refiere a los sujetos como individuos sino como personas o como seres humanos. Estos elementos no aparecen en Tu futuro en libertad, en el que la definición que aquí nos atañe se construye de una manera completamente distinta:

La sexualidad tiene que ver con la atracción, el placer y las preferencias sexuales; así como con la vinculación afectiva y erótica, el amor y la reproducción. Es importante mencionar que la sexualidad se experimenta o se expresa en forma de pensamientos, fantasías, deseos, creencias, valores, así como en actitudes y prácticas en las relaciones humanas. La sexualidad es el resultado de la interacción de factores biológicos, psicológicos, socioeconómicos, culturales, éticos y religiosos entre las personas (GDF, 2008: 9).

Notoriamente, en este fragmento no se usan apelativos como seres humanos ni se privilegian las funciones sociales que cumple la sexualidad. La atracción, el placer y las preferencias anteceden a los vínculos afectivos y eróticos, y en el discurso preceden al amor y la reproducción. A diferencia del libro de la Red Familia, en este la sexualidad se relaciona con la experimentación en varios niveles. Además, en el libro del GDF la sexualidad se concibe mediante la interacción entre un conjunto de factores, y no como una potencialidad intrínseca o naturalmente propia de las personas. 
b) ¿Cómo se ejerce la sexualidad? En los libros que se analizan en este artículo, el concepto de sexualidad permea las nociones sobre su ejercicio y sus implicaciones. En consecuencia, las distinciones entre ambos textos son visibles en todos los temas que abordan. Se ha señalado ya que en el discurso del GDF la sexualidad se entiende a partir del individuo, que implica la experimentación, y que no se expresa únicamente a través de prácticas sino de pensamientos, deseos y fantasías. Esta posición se mantiene en el apartado sobre relaciones sexuales:

El placer sexual es una dimensión sustancial de la identidad que fortalece la seguridad y la autoestima; es una aspiración positiva y enriquecedora de la vida humana. El placer puede ser emocionalmente muy intenso y producir sentimientos de felicidad; sobre todo cuando se experimenta ejerciendo los derechos humanos, cuando hay equidad y horizontalidad en las decisiones, cuando es producto de una decisión auto determinada y compartida, oportuna e informada, del acuerdo mutuo y no de la imposición o de una relación subordinada o asimétrica; cuando está libre de toda discriminación y violencia y de consecuencias no deseadas (GDF, 2008: 60).

En este fragmento se advierte que el individuo es el punto de partida para comprender la sexualidad. Una vez más, el énfasis se pone en sus efectos sobre la persona y no sobre el vínculo afectivo que se establece con la pareja sexual. De hecho, ese vínculo no se expone en términos de afectividad sino de derechos y de ciudadanía. Esto último resulta interesante, porque implica una noción específica sobre la sociedad, en la que la igualdad es un ideal tanto para la esfera pública como para la privada. En este discurso la sexualidad se concibe entonces como un ejercicio de experimentación entre personas iguales en búsqueda de su identidad, y no como un acto que necesariamente cumple una función.

El placer sexual se entiende de una forma muy distinta en Sexualidad y salud humana, donde se afirma que "[...] el amor juega un papel muy importante, pues da pleno sentido al placer erótico que el acto sexual proporciona" (Fernández, 2008: 19). Aunque la función reproductiva de la sexualidad se considera central en el discurso de la Red Familia, en el libro que aquí se analiza se reconoce que esta no es exclusiva. Desde esa perspectiva, la plenitud sexual resulta de un vínculo afectivo que se construye a través del amor, uno de los valores centrales del catolicismo.

La asociación entre acto sexual, amor y familia se mantiene en todo el texto, y su relación implícita con la función reproductiva conlleva una idea tradicional sobre el ejercicio de la sexualidad: "Quizá empezarás a sentir atracción hacia personas de otro sexo: las facciones de una cara, los movimientos del cuerpo, la sonrisa o la simpatía de algún amigo o amiga de pronto atraerán tu atención" (Fernández, 2008: 12). De este fragmento llaman la atención dos cosas: que las posibilidades de atracción parecen reducirse a parejas heterosexuales; y que está redactado en segunda persona para apelar directamente a lectoras y lectores. Volveremos sobre este punto más tarde. 
La concepción heteronormativa del acto sexual en el discurso de la Red Familia puede inferirse a partir de elementos visibles, tales como el argumento de que los sexos son complementarios. Pero es necesario atender también a los invisibles; lo que no se enuncia y lo que significa su ausencia. A diferencia de Tu futuro en libertad, en Sexualidad y salud humana no se incluye ninguna referencia a la diversidad sexual. Las parejas del mismo sexo no son adjetivadas como anómalas, sino que sencillamente no aparecen. Con ello se pretende naturalizar que las parejas sexuales están siempre conformadas por un hombre y una mujer.

La idea de la complementariedad entre lo masculino y lo femenino deriva del propio concepto de sexualidad, que en el discurso de la Red Familia cumple con la función de preservar la especie a partir de la fundación de la familia. Esta última se concibe como la unidad naturalmente constitutiva de la sociedad, cuya cohesión depende del amor. Así, en Sexualidad y salud humana las manifestaciones y prácticas que no se asocian con esta lógica se consideran conductas desviadas. La masturbación y la pornografía, por ejemplo, aparecen como signos de ansiedad, de angustia o de soledad. En el caso particular de la pornografía, esta se refiere como un delito en el que la objetivación de los seres humanos puede conducir a la perversión y a la violencia.

En el discurso del GDF, por el contrario, la sexualidad no tiene una función social sino individual. Además, los individuos se conciben como ciudadanos libres, responsables e iguales ante la ley. Se presume también que cuentan con información suficiente, por lo que la iniciación sexual no representa un problema siempre que sea una decisión consensuada. En esa premisa va implícita la idea de responsabilidad; el supuesto conocimiento sobre las consecuencias que puede acarrear el acto sexual conlleva una evaluación sobre los riesgos que involucra y las alternativas para evitarlos.

La noción de riesgo forma parte importante del discurso en los dos textos aquí referidos, en los que se advierte la posibilidad de sufrir abusos, de contraer infecciones o enfermedades de transmisión sexual, y de provocar un embarazo no deseado. En ambos casos, estas situaciones se construyen como peligros cuya prevención depende precisamente del acceso a una educación pertinente sobre el tema.

En ese orden de ideas, para prevenir el abuso se explican los derechos humanos a partir de referentes jurídicos. En los dos libros se recomienda denunciar cualquier violación a los derechos ante las autoridades estatales. Sin embargo, Sexualidad y salud humana introduce a la familia como mediadora entre los adolescentes y las instituciones jurídicas. Sobre los otros riesgos, en ambos textos se brinda información sobre los métodos anticonceptivos naturales y artificiales, su funcionamiento, sus ventajas y sus limitaciones. Empero, en el libro editado por la Red Familia se afirma que los métodos naturales son mejores, y se enfatiza la abstinencia como el único completamente confiable: "Los jóvenes no suelen arrepentirse de haber retrasado el inicio de las relaciones sexuales, en cambio sí se arrepienten de haberlas tenido tempranamente y en forma irresponsable" (Fernández, 2008: 21). 
Las discrepancias en torno a la forma de prevenir y solucionar los riesgos de una vida sexual activa son mucho más claras para el caso del embarazo no deseado. Tu futuro en libertad refiere la interrupción como una respuesta legítima y legal para las jóvenes que no desean la maternidad, introduciendo argumentos jurídicos, psicológicos, e, incluso, económicos: "Pero traer una hija o hijo al mundo supone una importante carga económica, que puede convertirse en un mecanismo de reproducción de la pobreza cuando no se tienen los recursos económicos para ella" (GDF, 2008: 97). Esta afirmación refleja el interés de las autoridades del Distrito Federal respecto de la educación sexual, un derecho que se convierte en asunto público porque sus consecuencias atañen a las instituciones estatales.

Sexualidad y salud humana, en cambio, mantiene una posición estrictamente apegada al valor de la vida, que emana de la doctrina católica. Al igual que en el caso de la diversidad sexual, la interrupción del embarazo no aparece como una alternativa porque no es mencionada en el libro. En cambio, la solución que se ofrece para las adolescentes preñadas es "[...] la posibilidad de darlo en adopción, a través de alguna de las instituciones debidamente autorizadas para hacerlo. Al nacer, ella decidirá libremente si lo conserva o si lo confía a una familia que le asegure un mejor futuro y garantice su educación [...]" (Fernández, 2008: 41).

c) ¿Quién tiene autoridad para orientar a los adolescentes sobre sexualidad? El ejercicio de la sexualidad durante la adolescencia se problematiza porque se asume que el tránsito entre la infancia y la adultez es especialmente complicado. Las trasformaciones físicas y psicológicas que se experimentan en esa fase repercuten en el desarrollo de la personalidad. Además de la inestabilidad emocional provocada por esos cambios, las y los adolescentes tienen poca experiencia y requieren de una adecuada orientación para su desarrollo. Este argumento, presente en ambos libros, justifica la necesidad de una educación sexual en la que las y los jóvenes cuenten con la guía de personas calificadas. Pero ¿quiénes son esas personas?

En Salud y sexualidad humana puede leerse que "la educación sexual corresponde en primer lugar a la familia, y debe estar de acuerdo con sus principios, costumbres y valores" (Fernández, 2008: 27). La aseveración se extiende también a la salud y la higiene. Por ejemplo, en un apartado en el que se explica el ciclo menstrual, se refiere la posibilidad de experimentar anomalías, en cuyo caso la joven "[...] deberá consultar a la mamá y en caso necesario al médico" (Fernández, 2008). Así, la importancia de la familia se enfatiza a través de una continua referencia a esta como mediadora entre adolescentes y otros actores sociales.

El rol de intermediación no aparece en Tu futuro en libertad. En este libro la familia se entiende como una institución en la que se desenvuelven los individuos, pero estos se consideran independientes en otros espacios: "Las principales fuentes de educación sexual son: la familia, la escuela, las instituciones de salud y desarrollo social, así como los medios de comunicación. Las iglesias también regulan la vida sexual de sus fieles, pero legalmente no pueden hacerlo 
en las instancias gubernamentales, porque vivimos en un estado laico" (GDF, 2008: 125). Desde esta perspectiva, la educación sexual compete a un abanico más amplio de instituciones, circunscritas en el marco de un Estado plural y liberal. El último enunciado del fragmento puede pensarse además como una respuesta a los debates por la aprobación de las leyes sobre sociedades de convivencia y la despenalización del aborto. Así pues, su propósito consiste en subrayar la laicidad del Estado para legitimar su autoridad por encima de las instituciones religiosas.

\section{La multiplicidad de voces como recurso pedagógico}

En sus estudios sobre la poesía y la novela, Bajtin (1989) señaló que la última constituye un género especialmente rico porque incluye múltiples voces que dan cuenta de la diversidad lingüística, cultural y social del contexto en el que se produce. El análisis que aquí nos ocupa no puede pensarse como una comparación entre estilos literarios, porque ambos son textos educativos. No obstante, también en estos es posible apreciar una multiplicidad de voces que contribuyen en la construcción del discurso. Es importante aclarar que el uso de varias voces no necesariamente refleja la pluralidad, pues en estos casos responde exclusivamente al objetivo de sustentar el argumento que las y los autores desarrollan en los libros de texto. Así pues, las identidades de quienes emiten sus opiniones (por ejemplo, en Tu futuro en libertad) o sus análisis profesionales (científicos, juristas y médicos, en ambos libros) no son relevantes sino en la medida en que fungen como un recurso pedagógico para "confirmar" la veracidad del discurso emitido por quienes escriben los libros de texto.

El uso de esas voces es muy similar en ambos textos. En ellos se recurre al discurso científico, al jurídico, al de los derechos humanos y al de igualdad de género. La única particularidad que presenta Tu futuro en libertad es que incluye además un discurso sobre la diversidad sexual, y que acude a citas extraídas de entrevistas a adolescentes. Por su parte, Sexualidad y salud humana presenta la característica distintiva de incluir un discurso moral.

Lo importante en este punto es destacar que, aunque en ambos textos se retoman prácticamente los mismos recursos discursivos, estos se utilizan para legitimar posiciones completamente distintas respecto de la sexualidad. En el libro editado por la Red Familia, por ejemplo, se hace una continua referencia a la ciencia para afirmar que existen investigaciones en las que se ha demostrado que los sexos masculino y femenino son complementarios por naturaleza. $T u$ futuro en libertad recurre exactamente a la misma estrategia para argumentar que no se han detectado los orígenes de la diversidad sexual y que, por lo tanto, no puede considerarse antinatural. El tema se vincula posteriormente con la homofobia y la discriminación, que en el libro se califican como prácticas indeseables en una sociedad democrática y regida por un Estado laico.

Otro ejemplo del uso del discurso científico para justificar la posición ideológica en ambos textos es el de la masturbación y el autoerotismo. En la doctrina católica la sexualidad está pensada como una actividad natural que cumple con una función reproductiva, o en todo caso co- 
municativa para garantizar la unión familiar. Las prácticas que no se ajustan a esas directrices resultan reprobables. Así, en Sexualidad y salud humana se afirma que "no existe evidencia médica sobre los daños físicos que esta práctica pueda ocasionar. Sin embargo, cuando se vuelve recurrente puede denotar ansiedad, estrés, exceso de estímulos visuales, angustia o sentimientos de soledad, situaciones que merecen ser atendidas y resueltas de fondo. Si tienes dudas, coméntalas con tus padres o alguien de tu confianza" (Fernández, 2008: 19). De este fragmento cabe rescatar dos cosas. En primer lugar, la referencia a la medicina se usa para legitimar un discurso basado en la creencia de que la masturbación denota problemas psicológicos, a pesar de que se reconoce que no existen evidencias científicas para respaldar ese argumento. Por otro lado, mediante el uso de la segunda persona del singular, se apela al lector o lectora. El propósito de este recurso consiste en formular un enunciado realizativo, ${ }^{9}$ en el que quien lee recibe una invitación para acercarse a personas con más experiencia. Igual que en otros casos, aquí los miembros de la familia se identifican como guías. Además, se propone que los familiares contribuyen a resolver problemas que las y los jóvenes no pueden enfrentar por sí mismos.

El contraste con el libro del GDF es evidente. En este las prácticas sexuales tienen que ver exclusivamente con el individuo y la exploración de su identidad:

Las investigaciones reportan que la masturbación no causa daño físico ni mental; al contrario, te ayuda a conocer tu cuerpo y en su caso a ensayar tu próxima vida sexual [...] Debido a los prejuicios en contra de la sexualidad se han creado una serie de falsas creencias acerca de la masturbación [...] No hagas caso a estas falsas creencias que no tienen base científica y sólo buscan inhibir las expresiones sanas y responsables de la sexualidad (GDF, 2008: 60-61).

Llama la atención que en este fragmento se usan exactamente los mismos recursos que en el citado anteriormente, pero para defender la posición contraria. Por un lado, el discurso científico pretende dar credibilidad a la afirmación de que el autoerotismo no es una conducta desviada ni causa daño. Por otro, se establece un vínculo con quien lee a través de un enunciado realizativo en el que se exhorta a la experimentación. Además, el énfasis se pone sobre el individuo y no sobre personas con más experiencia. El enunciado invita a rechazar posturas contrarias al discurso de Tu futuro en libertad, y se recurre otra vez a la ciencia para descalificarlas.

El uso del discurso jurídico tiene efectos muy similares. En ambos textos se retoma para referir los derechos de la ciudadanía, así como las responsabilidades del Estado para garantizarlos y para mantener el orden social. No obstante, el ideal de orden que se plantea en cada libro es también diferente. Sexualidad y salud humana construye su discurso a partir de una ideología

9 J. L. Austin (1977) clasifica los enunciados en constatativos y realizativos. Los primeros se refieren a aquellos que pretenden constatar un hecho y que por lo tanto pueden evaluarse a partir de un criterio de verdad; los segundos implican acciones, y su evaluación depende de las condiciones en las que se presentan y si son o no afortunados.

Diólo pos 
proveniente del catolicismo, en la que la familia tradicional se coloca en el centro de la organización social. Por ese motivo, en este se enfatizan las funciones reproductiva y afectiva de la sexualidad:

En sexto año de primaria estudiaste que cuando un hombre y una mujer se aman deciden compartir su vida y formar una familia. En nuestro país las leyes le dan formalidad a esa unión mediante el contrato del matrimonio civil. La legislación mexicana establece los derechos y las obligaciones de la pareja entre sí y hacia sus hijos. Así, el matrimonio es reconocido como la primera de las realidades sociales del ser humano en la que se funda la familia (Fernández, 2008: 30).

Mediante la afirmación de que la importancia del matrimonio se reconoce en la legislación mexicana, en el fragmento anterior el discurso jurídico se utiliza para legitimar la idea de que la familia se enarbola como la unidad básica a partir de la cual se deriva el tejido social. Pero cabe cuestionar y reflexionar en torno a las palabras que se usan para hacer tal afirmación. Supuestamente, el matrimonio es una realidad social del ser humano, y resulta del amor de un hombre $y$ una mujer que deciden no solo compartir su vida, sino además formar una familia.

El discurso jurídico para referir las responsabilidades del Estado es recurrente también en Tu futuro en libertad, en el que sin embargo no se habla de seres humanos sino de ciudadanos con derechos. El matrimonio se entiende entonces como un acto en el que se funda, no una familia sino un hogar, que goza de reconocimiento legal con independencia de la identidad sexual de sus miembros: "Este tipo de leyes se considera un primer paso hacia el reconocimiento pleno de la igualdad de derechos que deben tener las parejas del mismo sexo." (GDF, 2008: 53). Inmediatamente después se aborda la validez moral de la diversidad sexual, y luego se describe a la homofobia y la discriminación como problemas sociales.

De aquí se infiere que el discurso del GDF se basa en principios liberales que impulsan la igualdad ante la ley, y un Estado laico e incluyente en el que la igualdad de género no está en función del sexo biológico sino de la identidad. El esfuerzo por mostrar la política de inclusión se hace visible también mediante la introducción de fragmentos de entrevistas a adolescentes de la Ciudad de México, en los que expresan sus opiniones sobre su condición de jóvenes y sobre la sexualidad.

En términos generales, puede decirse que ambos libros construyen su discurso dando cabida a voces múltiples cuya función consiste en legitimarse. Del análisis precedente se concluye que los discursos científico, jurídico, de derechos humanos y de igualdad de género se utilizan de la misma forma en los dos textos: se incluyen para legitimar la posición que se sostiene en estos, sin ningún análisis o discusión para justificarla o para reconocer la validez de posiciones distintas. 
Sin embargo, es posible identificar dos diferencias sustantivas respecto de la introducción de discursos múltiples. Las ideas que se fundan en la laicidad del Estado están presentes únicamente en el libro editado por el GDF, en el que se usan para defender la autoridad del Estado por encima de otras instituciones sociales, entre las que se encuentran las organizaciones religiosas. Además, las voces de sujetos que pertenecen al mismo grupo etario al que va dirigido el texto se usan para dar una sensación de inclusión y de igualdad ante la ley, que comulga con el discurso liberal del GDF.

\section{El uso del lenguaje y el vínculo con el destinatario}

Como se ha dicho ya, en este documento se suscribe la propuesta de Peirce (1987) acerca del dinamismo en el proceso de significación y de la necesidad de ubicarse en el contexto del acto comunicativo para comprenderlo. En ese orden de ideas, los pronombres representan un problema analítico especialmente complejo, puesto que su significado cambia dependiendo de la situación en la que se presentan. Dicha dificultad fue señalada por Benveniste (1971), quien argumentó que estos no poseen un referente fijo sino que se construye durante el acto comunicativo. Por ejemplo, en los libros de educación sexual que aquí se analizan, los emisores del mensaje se construyen como un nosotros frente a un tú, que no es una persona específica sino todo aquel que se convierta en destinatario del mensaje a través de la lectura.

Aquí se sostiene que en los libros analizados el uso de pronombres como nosotros y tú constituye un recurso para mantener el vínculo con el destinatario o destinataria. Sin embargo, su uso cambia en función de lo que se dice y de la intención de generar una posición en quien lee el texto. En ambos casos pueden observarse tres formas de construir enunciados:

i) La información que aparece como neutral no suele ir acompañada de ningún pronombre, y en ocasiones se presenta con verbos reflexivos que carecen de todo vínculo con emisores y receptores. Enunciados como "la sexualidad es..." o "ser hombre o ser mujer se vincula con..." pueden clasificarse como constatativos en los términos planteados por J. Austin (1982), pues pretenden referir una realidad objetiva y pueden evaluarse a partir de un criterio de verdad.

La estructura de un enunciado en el que no se usan pronombres personales, le reviste de una supuesta neutralidad que reafirma las pretensiones de verdad del discurso (Foucault, 1977). En otras palabras, la ausencia de pronombres refuerza la idea de que lo que se dice es cierto y legítimo, especialmente si se menciona alguna autoridad discursiva. Decir que "un dato importante descubierto por la psicología, es que el modo masculino y el modo femenino de existir son complementarios no sólo entre los sexos, sino también en el interior de cada sexo" (Fernández, 2008: 33) parece más legítimo que afirmar que "encontramos así, una diversidad de actuaciones del ser mujer, del ser hombre, del 
relacionarse amorosamente del ser homosexual, del ser lesbiana, del ser heterosexual, del ser bisexual, del ser transexual [...]" (GDF, 2008: 51), en el que el uso implícito del pronombre nosotros genera la impresión de que el enunciado es más una opinión que un hecho. Los enunciados que carecen de pronombres explícitos e implícitos pueden encontrarse en ambos textos, y deben analizarse cuidadosamente, porque su pretendida objetividad suele estar cargada de posiciones ideológicas. Tal y como se expuso previamente, esas posiciones pueden identificarse a través de palabras que fungen como índices.

ii) Explícito o no, el pronombre nosotros aparece por lo general en enunciados que, además de información, presentan un componente normativo. El uso de este pronombre remite a la construcción del emisor como un colectivo en el que la posibilidad de incluir al lector permanece abierta. Por ejemplo, en el libro editado por el GDF puede leerse que "[...] al vivir la sexualidad de forma abierta, informada y responsable nos relacionamos afectivamente mejor y vivimos más libres y felices y podemos aportar lo mejor de nosotras y nosotros para mejorar nuestra ciudad" (GDF, 2008: 18). No queda claro si el pronombre nosotros tiene como referente al grupo de personas que se constituyen como emisores del discurso o si este incluye también a quien lee. En cualquier caso, el uso del pronombre nosotros modifica el vínculo con el lector; es más directo que en el caso descrito en el inciso anterior y personaliza el discurso. La posibilidad de incluir al lector en el pronombre nosotros remite, además, a la idea de que este comparte también las posiciones ideológicas que se expresan en el discurso.

iii) El pronombre tú se usa explícitamente en ambos libros para formar un vínculo directo con el destinatario. A diferencia de los dos casos anteriores, el uso del tú no se relaciona nunca con la construcción de enunciados constatativos sino realizativos. En el texto de los libros no pueden hacerse afirmaciones sobre el lector (tú); en cambio, pueden formularse interrogantes y exhortaciones a la reflexión o a la acción. En ese sentido, tú apela directamente a quien lee y tiene el objetivo de generar una reacción en este a través de preguntas, consejos o guías para la actividad cotidiana. Así, en Sexualidad y salud humana se enuncia: "Tienes derecho a decir NO a la actividad sexual precoz. Si decides no tener sexo hasta comprometerte en una unión estable como es el matrimonio, deberás esperar el momento adecuado hasta encontrar a la persona con quién compartir tu vida" (Fernández, 2008: 21). Si bien este tipo de enunciados no son órdenes, lo cierto es que la forma en la que se plantean apela directamente al lector y le obliga cuando menos a reflexionar sobre la pertinencia de actuar o no conforme a la posición que se manifiesta en el discurso. 
Una vez más, llama la atención que en ambos textos se recurre a maneras muy similares de construir el discurso y de establecer vínculos con el destinatario para incidir en sus posiciones de este respecto de la sexualidad. Sin embargo, en este punto puede observarse una distinción importante no solo en lo que se refiere a la ideología subyacente sino, incluso, en la frecuencia con la que se hace uso de los pronombres antes citados.

Explícitos o no, nosotros y tú son mucho más recurrentes en Tu futuro en libertad que en Sexualidad y salud humana. Esta diferencia se advierte desde el propio título de los libros, y se profundiza en la medida en que se desarrolla el discurso. De aquí se derivan dos efectos:

- El libro editado por el GDF mantiene un tono menos formal que el de la Red Familia, lo cual genera un lazo mucho más cercano con quienes leen. No debe soslayarse que los destinatarios clave de estos textos son adolescentes, y en ese sentido la informalidad puede resultar benéfica para atraer su atención.

- Adicionalmente, Tu futuro en libertad hace un mayor uso de los pronombres nosotros y tú cuyo efecto consiste en incentivar cierto tipo de actitudes entre los destinatarios. En ese sentido puede decirse que en ese libro se asume al lector como un sujeto mucho más activo y reflexivo que en el que edita la Red Familia. En este último el uso de tú se aplica sobre todo para ejercicios y para cuestionamientos sobre decisiones que el destinatario ha de resolver por sí mismo, pero en las que se le impulsa a consultar a familiares bajo la premisa de que estos tienen más experiencia.

\section{Conclusiones}

El objetivo en este texto consiste en comparar la construcción de los discursos en dos libros sobre educación sexual para adolescentes, uno editado por el Gobierno del Distrito Federal y el otro por la Red Familia. A partir de las dimensiones propuestas como ejes para la comparación, pueden extraerse las siguientes conclusiones:

1) Los discursos se produjeron bajo condiciones similares, puesto que los libros se editaron en el año 2008 tras la polémica suscitada en torno a la aprobación de la ley de sociedades de convivencia y la de despenalización del aborto. Esto implica que ambos discursos surgieron como respuesta a una misma preocupación, aunque la propuesta para solucionarla es radicalmente distinta. Esa diferencia puede explicarse por el hecho de que se fundan en esquemas de valores distintos.

2) Los libros comparten la mayoría de los temas que se consideran parte esencial de la educación sexual para adolescentes, pero el discurso en torno a ellos se articula de diferente forma en virtud de las posiciones ideológicas subyacentes: el GDF defiende una 
idea de orden social basada en el liberalismo, con un Estado laico capaz de garantizar los derechos de los ciudadanos y su igualdad ante la ley. En ese ideal el individuo se concibe como libre, autónomo y racional. Por su parte, la Red Familia impulsa un orden social en el que la familia desempeña un papel fundamental para el desarrollo de personas, y no de individuos, cuyos derechos han de estar garantizados por el Estado. Estas posturas, que a primera vista pueden parecer abstractas, son observables a través de la identificación de algunas palabras que fungen como índices de una carga ideológica en particular, así como de los enunciados que se construyen en torno a ellas. Así, en Tu futuro en libertad hay un uso recurrente de términos como individuo, libertad, derechos, responsabilidades, laicidad, diversidad, respeto y tolerancia, mientras que en Sexualidady salud humana se identifican conceptos como ser humano, familia, matrimonio, valores, libertad, derechos, responsabilidades y amor. Es viable comparar el discurso sobre la sexualidad a partir de tres temáticas:

a) La definición del concepto, que en el texto de la Red Familia se vincula con el amor, la comunicación, la reproducción y el placer mutuo, mientras que en el del GDF adquiere un carácter individual;

b) el ejercicio de la sexualidad, comprendido en Sexualidad y salud humana con base en los criterios heteronormativos que supone la familia tradicional, y en el que la experimentación se considera una actividad que denota trastornos psicológicos, a diferencia de Tu futuro en Libertad, en el que esta se define como parte de un proceso natural; y

c) las instituciones consideradas legítimas para orientar a los jóvenes sobre sexualidad, que en Sexualidad y salud humana se restringen a la familia y la escuela, mientras que Tu futuro en libertad considera también al Estado, las instituciones de salud, de desarrollo social, y los medios de comunicación.

3) En ambos libros se incorporan múltiples voces que contribuyen a legitimar el discurso. En los dos textos se recurre a la ciencia, a la legislación mexicana, a los derechos humanos y a la igualdad de género, para justificar la posición ideológica de cada uno. De aquí llama la atención que esos recursos se utilizan exactamente de la misma forma en ambos textos y responden a la misma necesidad; sin embargo, a través de estos se justifican posiciones esencialmente distintas y basadas en principios contrapuestos. Vale la pena recordar también que Tu futuro en libertad incorpora además el discurso de la laicidad y las voces de un grupo de adolescentes, en un intento por mostrar su congruencia con la inclusión y la diversidad, que aparecen a lo largo de todo su discurso.

4) En general, puede decirse que los dos libros están redactados: a) de manera neutral (es decir, sin pronombres personales), cuando lo que se dice se pretende objetivo y sin otro 
propósito que informar; $b$ ) con el pronombre nosotros, cuando se busca una redacción menos formal, en la que se procura un vínculo de más confianza con el lector, y en la que el emisor se construye como un colectivo en el que la posibilidad de incluir al destinatario permanece abierta; y c) con el pronombre tú, en las partes en las que la apelación al lector resulta fundamental puesto que se le invita a identificarse con determinadas situaciones, a reflexionar o a actuar. La primera forma de redacción es ampliamente utilizada por ambos libros; la segunda se presenta en ambos, pero es más recurrente en Tu futuro en libertad; $y$ la tercera se utiliza en el libro de la Red Familia, sobre todo para señalar la necesidad de reflexión y de orientación familiar, mientras que en el del GDF promueve la experimentación y la acción del individuo:

Las páginas que siguen hablan de libertad, de igualdad y equidad entre mujeres y hombres; de toleran$\underline{\text { cia }}$, del respeto a nuestros sentimientos y deseos hacia quienes son diferentes o no piensan como tú. De lo que sientes y quieres, de lo que a veces te quema el alma. Con este texto, el Gobierno de nuestra ciudad quiere presentarte una propuesta distinta de educación, una propuesta que incluye la invitación a participar en la construcción de una ciudad con equidad de género. [...] (GDF, 2008: 2).

En ambos libros pueden identificarse enunciados tanto constatativos como realizativos. Los primeros se utilizan para brindar información supuestamente objetiva y susceptible de contrastación a partir de un criterio de verdad. Los segundos corresponden a partes del discurso en las que el destinatario es cuestionado o se le invita a reflexionar, a experimentar o a actuar, y son más utilizados en Tu futuro en libertad.

Los discursos en los libros aquí analizados corresponden a textos educativos, y como tal se presentan como puramente informativos y, por lo tanto, como neutrales. Sin embargo, a través del análisis de estos es posible argumentar que tanto Sexualidad y salud humana como Tu futuro en libertad se construyen con base en principios específicos y nociones claras sobre el orden social; el primero parte de valores tradicionales y de la familia como unidad social básica, y el segundo, de valores liberales y del individuo como centro. En ese sentido, ambos poseen un carácter normativo que se vuelve evidente, sobre todo en los enunciados realizativos en los que se usa el pronombre tú, y cuyo objetivo consiste en generar una posición ideológica entre los destinatarios.

Es importante subrayar que la educación sexual resulta trascendente para los dos grupos que publican los libros analizados a lo largo de este texto, porque en el ejercicio de la sexualidad se manifiestan nociones en mayor o menor medida compatibles con el ideal de sociedad que plantea cada uno. En ese orden de ideas, aquí se sostiene que, con independencia de que sus discursos estén anclados en valores liberales o tradicionales, en los dos libros se pretende ejercer 
cierto control sobre los destinatarios puesto que en ellos se ofrecen parámetros de lo que es aceptable o no, así como de lo que debe reflexionarse, discutirse, e incluso enunciarse.

\section{Referencias bibliográficas}

Austin, J. L. (1982). Cómo hacer cosas con palabras: Palabras y acciones. Barcelona: Paidós. Bajtin, M. (1989). Teoría y estética de la novela: Trabajos de investigación. Madrid: Taurus.

Benveniste, E. (1971). Problemas de lingüística general. México: Siglo XXI.

Blancarte, R. (2008). Para entender el Estado laico. México: Nostra Ediciones.

Fernández, P. (2008). Sexualidad y salud humana. México: Santillana.

Foucault, M. (1977). Historia de la sexualidad. México: Siglo XXI.

INEGI (2013). Población, hogares y vivienda. http://www.inegi.gob.mx/

Irvine, J.; S. Gal (1995). Language Ideology and Linguistic Differentiation. En Kroskrity, P., Regimes of Language. Ideologies, Politics and Identities. Nuevo Mexico: School of American Research Press.

Levinson, S. (1983). Pragmatics. Cambridge: Cambridge University Press.

Perice, Ch. (1987). Obra Lógico - Semiótica. Madrid: Taurus.

Red Familia (2012). Principios rectores. http://www.redfamilia.org/ 